\title{
Transpiration-based micropump for delivering continuous ultra-low flow rates
}

\author{
Vijay Namasivayam ${ }^{1,3}$, Ronald G Larson ${ }^{1}$, David T Burke ${ }^{2}$ \\ and Mark A Burns ${ }^{1}$ \\ ${ }^{1}$ Department of Chemical Engineering, The University of Michigan, Ann Arbor, \\ MI 48109-2136, USA \\ 2 Department of Human Genetics, The University of Michigan, Ann Arbor, \\ MI 48109-2136, USA \\ ${ }^{3}$ Department of Electrical Engineering and Computer Science, The University of Michigan, \\ Ann Arbor, MI 48109-2136, USA \\ E-mail: maburns@umich.edu
}

Received 27 March 2002, in final form 26 September 2002

Published 17 January 2003

Online at stacks.iop.org/JMM/13/261

\begin{abstract}
In this paper we describe the design, construction and operation of a micropump that delivers continuous, ultra-low flow velocities at $\sim 100 \mu \mathrm{m} \mathrm{s}^{-1}$. The pumping concept is based on the commonly observed phenomenon of transpiration in plant leaves. A liquid meniscus is pinned inside a microchannel by selective hydrophobic patterning and the evaporation rate of the liquid at the meniscus is controlled. The controlled evaporative flux results in a regulated flow of the liquid from a reservoir to the meniscus. Using this technique, precise flow control $\left(5 \mathrm{nl} \mathrm{min}{ }^{-1}\right)$ has been achieved in several channel geometries for extended periods of time $(\sim 2 \mathrm{~h})$. Various factors affecting the performance of the pump were studied and theoretical predictions along with experimental results are presented. Such a micropump could find applications in emerging biological assays such as single-molecule studies of DNA and cell adhesion analyses.
\end{abstract}

(Some figures in this article are in colour only in the electronic version)

\section{Introduction}

The concept of miniaturization of chemical analysis systems or 'Lab-on-a-chip' has witnessed significant developments over the last decade. The power of a Micro-Total Analysis System ( $\mu$ TAS) is especially apparent in areas of biological assays such as DNA sequencing (Liu et al 1999, SalasSolano et al 2000), genotyping (Woolley et al 1997) and cell sorting (Fu et al 1999). As the push toward miniaturization continues, sample volumes are growing considerably smaller from microliters to nanoliters, and more components are being integrated onto the system. Several works emphasizing this trend of miniaturization and integration have been published in the last few years (Waters et al 1998, Burns et al 1998). This need for miniaturization and integration of fluidic components such as pumps and valves is also becoming apparent in emerging research areas like single-molecule assays and cellbased studies.
Single-molecule studies such as stretching of DNA molecules (Perkins et al 1995), functioning of molecular motors (Mehta et al 1999), transcription by RNA polymerase (Davenport et al 2000) are currently hot topics of interest in several research labs. DNA-based single-molecule studies have opened doors for alternate methods to map and sequence the genome. New genome mapping techniques based on endonuclease digestion on elongated and fluidfixed DNA molecules have been reported (Jing et al 1998). Sequencing at a single molecule level based on exonuclease digestion of stretched DNA molecules has also been proposed (Dorre 1997). All these new mapping and sequencing techniques rely on the ability to stretch DNA molecules. One single-molecule study showed that DNA molecules can be stretched in liquid-flow-based systems, where $\lambda$ DNA molecules were held at one end by an optical tweezer and an extensional flow caused the molecules to stretch (Perkins et al 1995). Such experiments require a 
pump to deliver continuous, ultra-low-velocity liquid flow rates.

Cell-based studies often require a pumping system that can generate focused, continuous, low-velocity flows. A recent study reported the use of multiple laminar streams in a microfluidic channel to deliver membrane-permeable molecules to selected subcellular microdomains (Takayama et al 2001). This technique was applied to study the subcellular processes of mitochondrial movement and changes in cytoskeletal structure. Studies like these are useful for noninvasively visualizing, probing and manipulating the cellular metabolic and structural machinery.

In medical diagnostics, microdialysis systems used for continuous monitoring of glucose levels represent a promising means to reduce the risk of late complications and improve the quality of life of diabetic patients. These systems also require a micropump to deliver very small flow rates in the order of

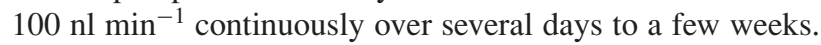
Important constraints of a microdialysis system include small and constant flow rates, small size and low weight, low energy consumption, simple construction and robust operation.

Silicon-based microfabrication technology has revolutionized the field of miniaturized chemical analysis systems and holds the potential for building micropumps for the specific applications discussed thus far. Several types of valved pumps employing thermopneumatic (Vand De Pol et al 1989), piezoelectric (Carrozza et al 1995), electrostatic (Zengerle et al 1992) actuation techniques and valveless pumps using electroosmotic (Manz et al 1995) techniques have been constructed on both silicon and glass substrates. However, most existing valved micropumps deliver pulsatile flows at relatively high velocities $\left(1 \mathrm{~mm} \mathrm{~s}^{-1}\right)$ and valveless electroosmotic pumps require the application of very high voltages $(\mathrm{kV})$ with very little room for flow control. Other kinds of valveless pumps that are based on diffuser/nozzle designs also deliver pulsatile flows (Olsson et al 1997).

The focus of this paper is on building a micropump based on earlier works (Namasivayam et al 2000, Goedecke and Manz 2001) for studies such as DNA stretching, glucose monitoring and subcellular labeling. A transpirationbased valveless micropump that works on the principle of evaporation from a liquid meniscus inside a capillary is presented for delivering the required non-pulsatile, ultralow flow rates. The paper discusses a theoretical basis for the pumping mechanism and includes a model describing the effect of the various control factors on the pumping velocity. The pumping mechanism is tested using devices constructed from silicon/glass and the experimental results are presented.

\section{Micropump design and modeling}

Our micropump design is based on the concept of transpiration at leaf surfaces. Transpiration is the phenomenon by which water is lost as water vapor through the pores (stomata) on the leaf surface. To compensate for the evaporative loss of water vapor, fresh water from the roots reaches the leaf surface (mainly by capillary action) (Canny 1998). In our transpiration-based micropump design, a liquid meniscus is pinned inside a microchannel by selective hydrophobic patterning (Handique et al 2000) and the evaporation rate of the liquid at the meniscus is controlled. Figure 1(a) shows a steady-state liquid meniscus pinned inside a slit-type microchannel. When the liquid is heated at the meniscus, the vapor pressure increases resulting in enhanced evaporation (Smith and Van Ness 1987). As the vapor diffuses out, a fresh liquid supply is drawn into the channel from a reservoir to sustain steady-state operation. The capillary-force-aided imbibition process continues until the reservoir is depleted after which the meniscus begins to recede.

Consider a pumping device as shown in figure $1(b)$, where liquid from the reservoir flows into channel $\mathrm{A}$ and gets pinned inside the channel (due to hydrophobic treatment). When the liquid at the meniscus is heated, the vapor diffuses out to channel B over a length $(L)$ and is driven out of the system by airflow in channel B. To match this flux loss from the meniscus, liquid from the reservoir flows into channel A, thus creating a continuous flow. The pumping action is entirely limited by the evaporation rate of the liquid at the meniscus and the diffusion rates of the water vapor away from the meniscus. In essence, the following parameters contribute to the pumping rate: temperature at the meniscus, the airflow rate, the distance of the liquid meniscus from channel $\mathrm{B}(L)$ and the ratio of cross-sectional areas in channels $\mathrm{A}$ and $\mathrm{B}\left(A_{E} / A_{F}\right)$.

In this section, we present a model that was developed with the objective of estimating liquid flow velocities that can be generated with the micropump. The model is also useful for understanding the influence of various control factors on the pumping velocities. Our model is built around equating the rate of evaporation from the meniscus with the liquid flow rate in channel $\mathrm{A}$. The evaporation rate $R_{E}$ is given by the expression

$$
R_{E}=M A_{E} N_{E}
$$

where $M$ is the molecular weight of the vapor, $A_{E}$ is the crosssectional area of the evaporation section and $N_{E}$ is the flux (moles/area/time). The flow rate of the liquid $\left(R_{F}\right)$ from the reservoir to the meniscus matches this evaporation rate as long as the resistance due to pressure drop is not pronounced,

$$
R_{F}=\rho_{l}\langle v\rangle A_{F}
$$

where $\rho_{l}$ is the liquid density, $\langle v\rangle$ is the average flow velocity and $A_{F}$ the flow cross-sectional area. The average velocity of the liquid flow can be extracted out by equating the two rates:

$$
\langle v\rangle=\frac{M A_{E} N_{E}}{\rho_{l} A_{F}} .
$$

Evaporation from a pinned meniscus within a microchannel occurs at two regions; a thicker intrinsic meniscus region controlled by capillarity and a thin film (lubrication) region controlled by disjoining pressure and capillarity. In completely wetting systems, the effect of disjoining pressure in the thin film region has a substantial effect on the rate of evaporation (Schonberg et al 1995). In our system since the meniscus is pinned in a hydrophobic (non-wetting) region, the effect of the extended meniscus (lubrication region) is neglected. The meniscus is also assumed to retain a flat shape since, with our hydrophobic patterning technique, we can obtain contact angles around 
(a)

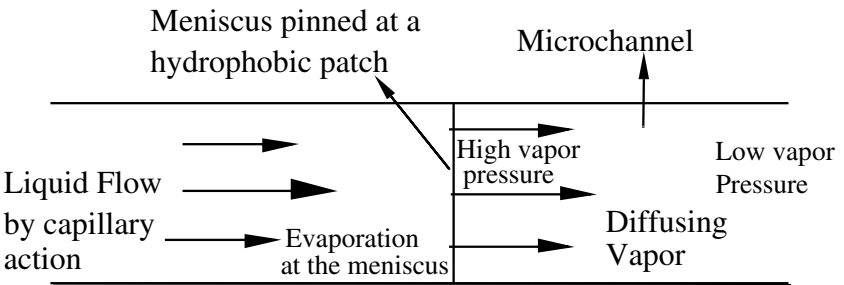

Heated Substrate

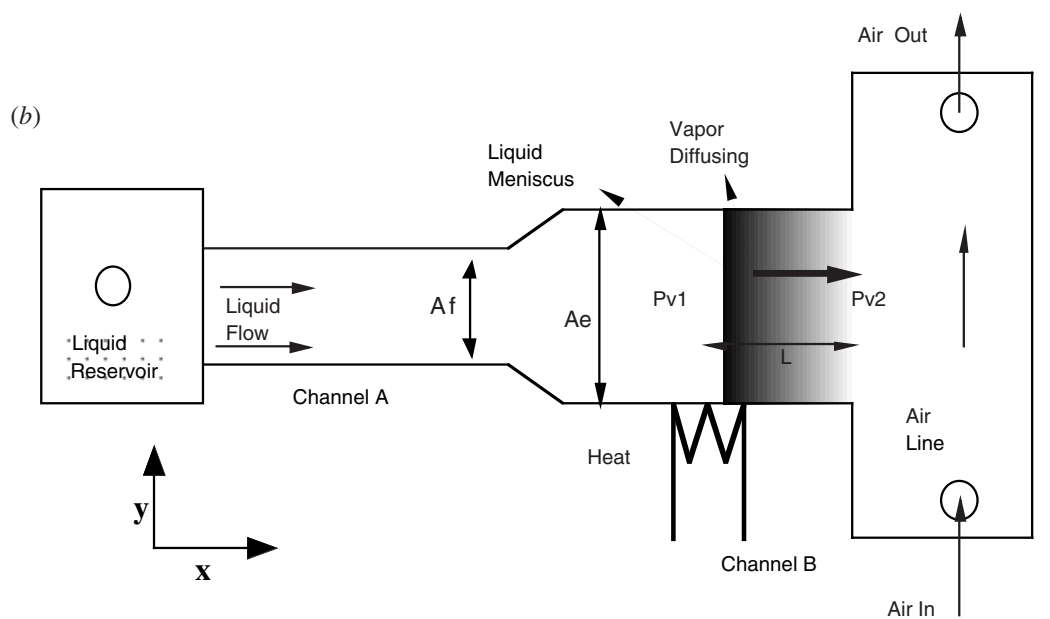

Figure 1. Micropump concept. (a) Schematic of working principle of transpiration-based micropump, $(b)$ complete device schematic illustrating the control factors and design parameters.

$90^{\circ}-100^{\circ}$ for water. The evaporative flux, $N_{E}$, is given by the expression

$$
N_{E}=D \frac{\partial \mathrm{C}_{v}}{\partial x}=\frac{D}{R T} \frac{\partial P_{v}}{\partial x}
$$

where $D$ is the diffusivity of the vapor, and $C_{v}$ and $P_{v}$ are the concentration and partial pressure of the vapor (Bird et al 1960). To determine the vapor pressure gradient $\nabla p$ at the meniscus (at $x=0$ ), we start from the basic equation for mass transfer of the vapor across the meniscus,

$$
D \nabla^{2} p=\frac{\partial p}{\partial t}+u \cdot \nabla p
$$

where $D$ is the diffusivity of the vapor, $p$ is the partial pressure and $u$ is the velocity of airflow in channel B. Under steadystate conditions, the accumulation term $\frac{\partial p}{\partial t}$ is equal to zero. The convective term $u \cdot \nabla p$ in equation (5) is included to describe the influence of airflow rate in channel B. The airflow rate in channel B not only helps maintain a constant vapor pressure, but also serves as an important control factor for convective mass transfer.

To solve equation (5) we assume the following boundary conditions:

- at $x=0$ (at the meniscus), $p=p_{v 1}$,

- at $x=L$ (in channel B), $p=p_{v 2}$,

where $p_{v 1}$ and $p_{v 2}$ represent the partial pressures of the vapor at the two ends of the design length $(L)$. For a micropump designed for pumping water, $p_{v 2}$ can be approximated to partial pressure of water vapor in the air pumped through channel B. In our case, the experiments were carried out when the relative humidity in the air stream was $40 \%$.

Equation (5) is first converted to a dimensionless form

$$
\frac{\partial^{2} P}{\partial X^{2}}+\frac{\partial^{2} P}{\partial Y^{2}}=P e U\left(\frac{\partial P}{\partial X}+\frac{\partial P}{\partial Y}\right)
$$

where

$$
\begin{gathered}
X=\frac{x}{L}, \quad Y=\frac{y}{L}, \quad U=\frac{u}{u_{0}} \\
P=\frac{p-p_{v 2}}{p_{v 1}-p_{v 2}}, \quad P e=\frac{u_{0} L}{D}
\end{gathered}
$$

with the following boundary conditions:

$$
\text { at } X=0, \quad P=1 \quad \text { and } \quad \text { at } X=1, \quad P=0 .
$$

Since the Peclet number for this system is greater than 1 , equation (6) can be solved using standard boundary layer approximations to obtain a rough estimate for the pressure profile (Cussler 1997). From the solution for pressure distribution, the average vapor flux at the meniscus is then obtained as

$$
\frac{\partial p}{\partial x} \mid=\left(\frac{P_{v 1}-P_{v 2}}{L}\right)\left(1+\sqrt{\frac{P e W}{L}}\right)
$$

where $L$ is the interface distance, $W$ is the width of the pinned meniscus and $P e$ is the Peclet number, a measure of the airflow velocity in channel $\mathrm{B}$. This expression for the pressure gradient 
(i)

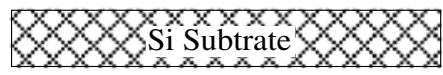

Silicon Oxide $\mid$ Grow Silicon Oxide $(0.2 \mu \mathrm{m}) \backslash$

(ii)

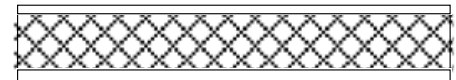

Spin Photoresist,

\& \& Development

(iii)

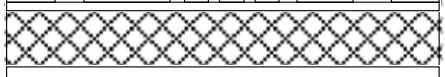

Evaporate Ti/Pt

$(10 / 200 \mathrm{~nm})$

(iv)
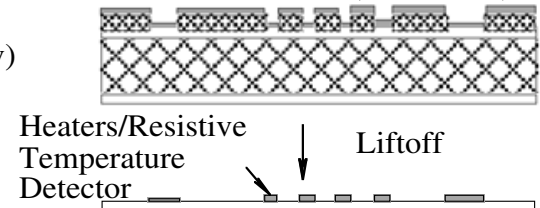

(v)
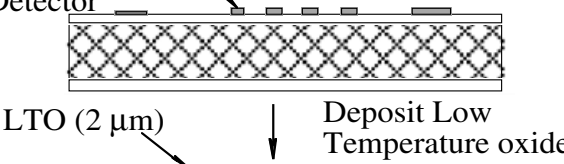

(vi)

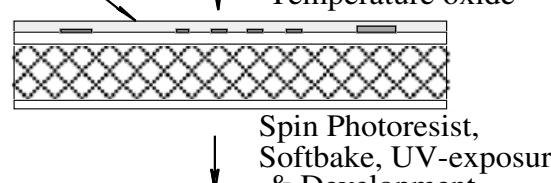

(vii)
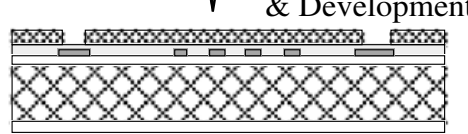

Etch metal,

$\checkmark$ Strip Photoresist

(viii)
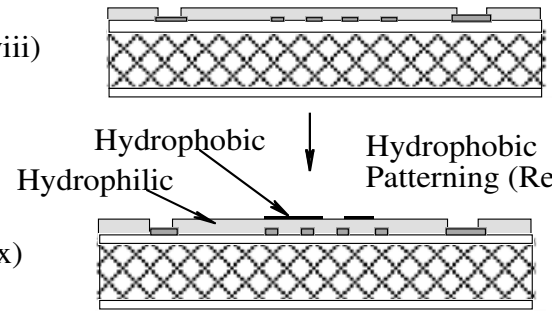

(a) (i)

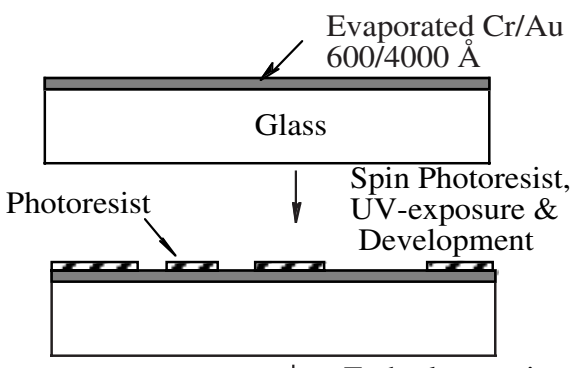

(iii)

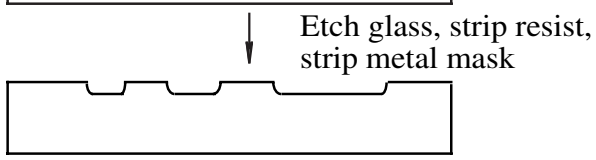

(iv)

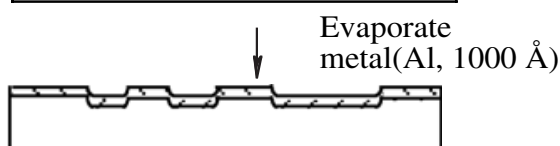

(v)
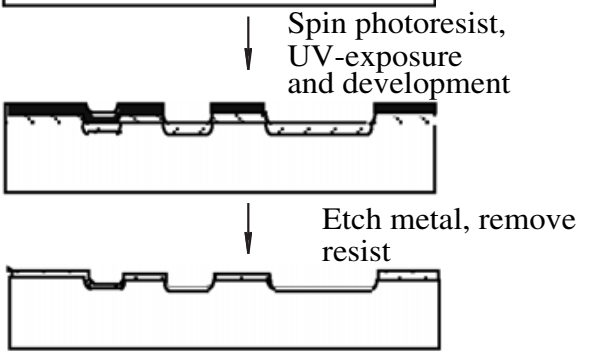

(vi)

(vii)
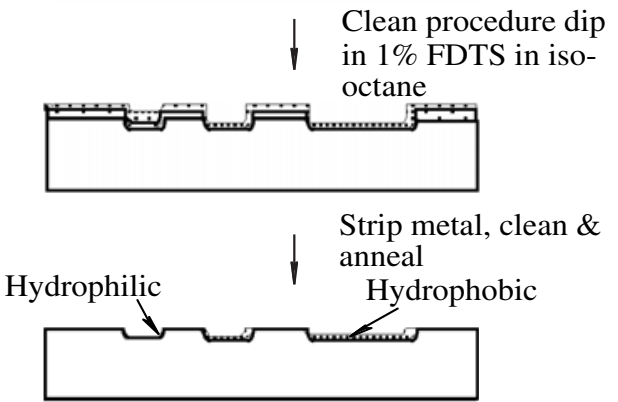

(b)

Figure 2. Schematic of process used for construction of the micropump. (a) Silicon side with heaters, temperature sensors and a hydrophobic patch, $(b)$ glass side with etched channel and a hydrophobic patch.

is incorporated in equation (3) to obtain the final expression for estimating the liquid pumping velocities:

$$
\langle v\rangle=\frac{M D}{\rho_{l} R T} \frac{A_{E}}{A_{F}}\left(\frac{P_{v 1}-P_{v 2}}{L}\right)\left(1+\sqrt{\frac{P e W}{L}}\right) .
$$

Pumping velocities estimated using this model are presented in section 4. Comparison of the model-estimated velocities with the experimental values shows a close fit.

\section{Materials and methods}

\subsection{Device construction}

The micropump is a silicon-glass hybrid system with microchannels etched into the glass side and heaters, and temperature sensors microfabricated on the silicon side. Hydrophobic patches are formed selectively over the required regions on both silicon and glass sides by a silanization procedure (Handique et al 2000). Detailed fabrication steps involved in the construction of the micropump are described below.

3.1.1. Silicon side fabrication: heaters, RTDs and hydrophobic patches. Figure 2 shows the simplified process flow for constructing the micropump. Device fabrication begins by constructing heaters and temperature sensors on the silicon side. A silicon wafer (p-type, $18-22 \Omega \mathrm{cm}$, boron concentration $\approx 10^{15} \mathrm{~cm}^{3}$ ) is used as a substrate for the growth of $\mathrm{SiO}_{2}$ thermal oxide. A $0.2 \mu \mathrm{m}$ thick layer of thermal oxide is grown on the silicon wafer (figure 2(a.i)) prior to metal 
deposition (figure 2(a.ii)). A positive photoresist is applied, patterned and developed (figure 2(a.iii)). A $0.2 \mu \mathrm{m}$ thick film of platinum is deposited on the substrate by electron beam evaporation (figure 2(a.iv)). A $0.01 \mu \mathrm{m}$ thick titanium metal layer (electron beam deposited) is used as an adhesion layer. The resist and the overlying metal layers are then lifted off by a development using Microposit 1112A remover in the solution (Shipley Co.). The platinum metal forms the heater and resistive temperature detector (figure 2(a.v)). Next, $2 \mu \mathrm{m}$ of low temperature oxide (LTO) of silicon is deposited (figure 2(a.vi)) to act as the barrier layer and the hydrophilic substrate. A second lithography is carried out and the LTO is etched in buffered hydrofluoric acid to open contacts with the metal contact pads (figure 2(a.viii)).

Specific regions on the silicon oxide (LTO) were rendered hydrophobic using a selective hydrophobic patterning technique developed in our research group. This technique involves patterning a thin film of $\mathrm{Al}$ on the wafer (that acts as a mask) and dipping the wafer in a silane solution (fluorododecyltrichlorosilane, FDTS $1 \mathrm{wt} \%$ ) in a nitrogen atmosphere. The exposed areas are rendered hydrophobic and the $\mathrm{Al}$ mask is removed by the wet etching process using a commercial $\mathrm{Al}$ etchant.

3.1.2. Glass side fabrication: channels, hydrophobic patches. Channel fabrication begins by depositing a $0.4 \mu \mathrm{m}$ metallic layer of gold (electron beam deposition) on the surface of a $500 \mu \mathrm{m}$ thick glass wafer (Dow Corning 7740). A $0.06 \mu \mathrm{m}$ layer of chromium was used as the adhesion layer. Photoresist (1827, $2 \mathrm{k}$ ) was applied and patterned using a glass channel mask and was developed. The metal layers were etched in a gold etchant (Gold Etchant TFA, Transene Co.) and a chromium etchant (CR-14, Cyantek Inc.). The accessible glass was then etched in a freshly prepared solution of hydrofluoric and nitric acid $(7: 3, \mathrm{v} / \mathrm{v})$. The etch rate was approximately $6 \mu \mathrm{m} \mathrm{min}^{-1}$ and the etch depth was measured using a surface profilometer. The resulting glass channel cross section had a trapezoidal shape due to the under etch. (A $50 \mu \mathrm{m}$ glass channel designed for $500 \mu \mathrm{m}$ wide rectangular cross section resulted in trapezoidal shape with $700 \mu \mathrm{m}$ at the top, $500 \mu \mathrm{m}$ at the bottom.) The metal layers were removed and the wafer was rinsed in DI water, then air-dried and oven-dried at $100{ }^{\circ} \mathrm{C}$ for $20 \mathrm{~min}$. Hydrophobic patterning was done on selected regions on the glass side using the same technique as described for the silicon side but a thicker photoresist (AZ 4620) was used in this case. The samples were then rinsed in DI water and air-dried. For the devices with the inlet from the top, holes were drilled by electrochemical discharge drilling (Shoji and Esahi 1990).

3.1.3. Device assembly. The glass side was then aligned on top of the silicon side and then bonded together using an optical adhesive (SK-9 Lens Bond, Sumers Laboratories, Fort Washington, PA). The bond was cured under an ultraviolet light source $(365 \mathrm{~nm})$ for $24 \mathrm{~h}$. To deliver a constant airflow into the device, a stainless steel hypodermic tubing is glued to the air-line hole using a fast-setting epoxy. A small teflon tubing connects the stainless tubing to an air pressure source and a pressure regulator.

\subsection{Instrumentation}

Precise temperature control $\left( \pm 0.1{ }^{\circ} \mathrm{C}\right)$ at the meniscus is achieved by using on-chip heaters and temperature detectors (RTDs). The chip was mounted on a PC board and interfaced with a Labview program (National Instruments). A software control system on the Labview program was used to control the temperature of the heaters. The airflow rate in channel B was controlled at a specific rate using an external air source and a pressure regulator (Matheson Gas Products Inc., Model 3701). The velocity of airflow was back-calculated by knowing the channel dimensions and the pressure applied.

\subsection{Velocity measurements}

Flow visualization was done by introducing fluorescein microspheres (fluoresbrite carboxylate microspheres, $3 \mu \mathrm{m}$ diameter, obtained from Polysciences Inc., PA) as tracer particles and observing them through a stereoscope (Model SZH 10, Olympus, Japan) and the results were recorded using a Hammamatsu SIT camera. Figure 3(a) shows the schematic illustrating the velocimetry technique and figure $3(b)$ shows the picture of the channel, where the liquid (DI water) mixed with fluorescent microspheres is pumped from the reservoir end to the meniscus. Centerline velocity of the fluorescent spheres was calculated by measuring the time taken by the spheres to travel between two reference (heater) lines in the device $1 \mathrm{~mm}$ apart. The average liquid velocity was estimated from the centerline peak velocity of the fluorescein spheres as given by the following expression:

$$
\langle V\rangle=\frac{2}{3} V_{\max } \quad \text { (for a rectangular channel). }
$$

Note that, in order to take into account the diffusion of the microspheres in the liquid, the distance traversed by the microspheres due to pure diffusion at room temperature was measured and this distance $(\sim 3 \mu \mathrm{m})$ was subtracted during velocity calculations.

\section{Results and discussions}

As explained earlier, the pumping velocity depends on the evaporation rate at the meniscus, which in turn depends on the following factors: the temperature at the meniscus $(T)$, the airflow rate in channel $\mathrm{B}$ and design parameters (ratio of cross sections $A_{E} / A_{F}$, interface distance $L$ ). Performance of the micropump was characterized under the influence of several control factors and the results are presented below.

\subsection{Model predictions and device operation}

Figure 4 shows the characteristics of the micropump, as predicted by the model described earlier (equation (8)) Figure 4(a) shows the range of pumping velocities that can be obtained by changing temperatures in various device geometries under a constant airflow rate of $10 \mathrm{~cm} \mathrm{~s}^{-1}$. Figure $4(b)$ shows the influence of temperature and airflow rate with the device geometry held constant. From the model, it is apparent that small flow velocities in the order of $100 \mu \mathrm{m} \mathrm{s}^{-1}$ can be achieved by the appropriate selection of channel dimensions. Theoretically, a continuous range of fluid velocities from near zero to $200 \mu \mathrm{m} \mathrm{s}^{-1}$ can be obtained 

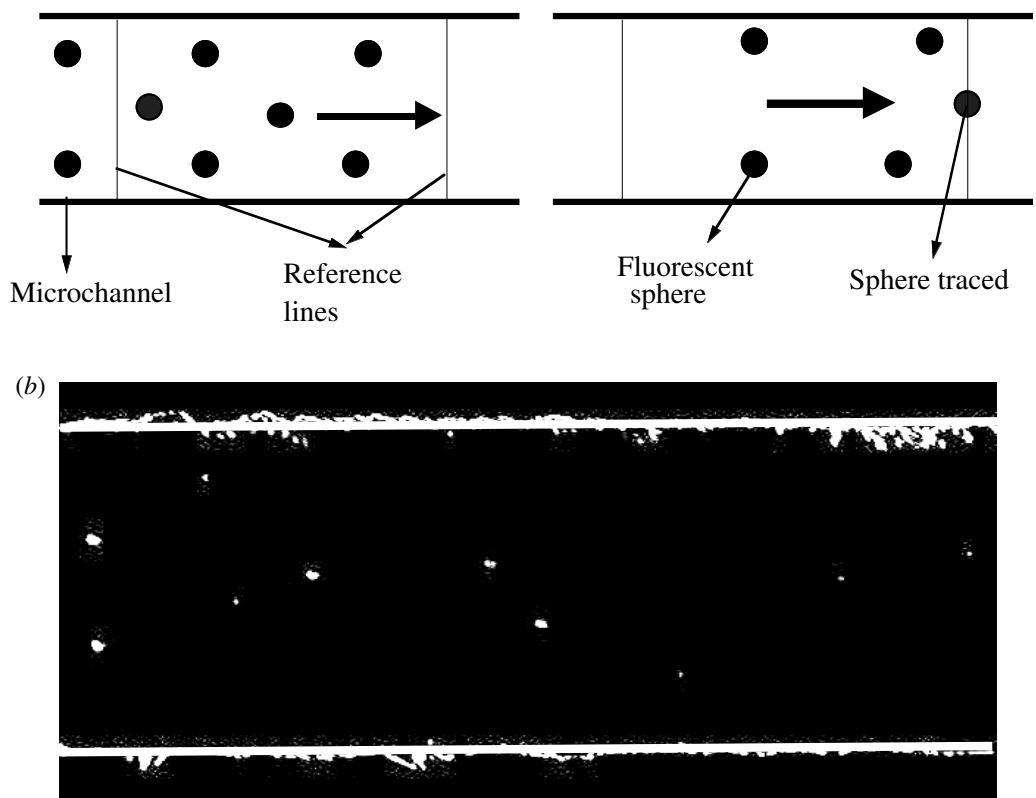

Figure 3. Particle velocimetry for estimating flow velocities. (a) Illustration of the velocimetry technique. Centerline velocities of the microspheres are measured to calculate the average liquid velocities. (b) Photograph of the microfluidic channel with fluorescent spheres used for velocimetry.

by merely changing the temperature at the meniscus from room temperature to $90{ }^{\circ} \mathrm{C}$. Device geometry also serves as an in-built control parameter for obtaining the required flow velocities. Based on these estimates from the model, different micropump designs with varying channel geometric ratios were fabricated and tested.

Figure 5(a) shows the photograph of a micropump constructed for this study. To operate the system, liquid is introduced at the reservoir, which enters the channel by capillary action and stops at the hydrophobic regions. To facilitate a constant supply of liquid to the meniscus, a small pressure head ( $2^{\prime \prime}$ of water) is maintained at the reservoir end. Figure 5(b) shows the zoomed-in picture of the device, where the liquid (DI water) is stopped at the hydrophobic patch. The shape of the meniscus stopped on the hydrophobic region is seen to be flat. (The static contact angle of water on the surface of a silicon wafer before and after the hydrophobic treatment was measured to be $30^{\circ} \pm 0.5^{\circ}$ and $100^{\circ} \pm 0.5^{\circ}$, respectively). The hydrophobic patches were also found to be durable under the influence of different solvents, acids and bases (Handique et al 2000). The stability of these hydrophobic films under high temperatures is usually of concern. In our case, the films have to withstand temperatures up to $100{ }^{\circ} \mathrm{C}$ during operation, since all other assembly and testing steps are carried out at low temperatures. Srinivasan et al (1997) have reported FDTS film to be stable up to $400{ }^{\circ} \mathrm{C}$ in air for 5 min.

\subsection{Control factors}

4.2.1. Temperature. Temperature is perhaps the most important control factor of the micropump. To characterize the effect of temperature on the pumping velocity, DI water mixed with fluorescent microspheres was stopped at the meniscus by a hydrophobic patch and the heater just behind the meniscus was turned on. Temperature at the meniscus was gradually increased using the LabView controller and the pumping velocity was estimated for each temperature setting. The experiment was repeated in another device with a different cross-sectional ratio $\left(A_{E} / A_{F}\right)$. Airflow rate was maintained constant during the course of the experiment. Figure 6 shows the estimated average flow velocities at several operating temperatures of the heater near the meniscus in two different device geometries. The experimentally observed values fit closely with the predicted values from the model as shown in figure 6.

4.2.2. Airflow. The influence of airflow was studied in a device with a cross-sectional ratio $\left(A_{E} / A_{F}\right)$ of 5 . Velocity measurements were made at various operating temperatures under two different airflow velocities $\left(10\right.$ and $\left.20 \mathrm{~cm} \mathrm{~s}^{-1}\right)$ in channel B. Figure 7 shows the variation of liquid flow velocity in channel A with changes in the airflow rate in channel B. Increasing the airflow rate results in enhanced diffusion of vapor from the channel and hence leads to increased liquid flow rates in channel A. However, higher flow rates of air $\left(>25 \mathrm{~cm} \mathrm{~s}^{-1}\right)$ would cause the pinned meniscus to recede. This is mainly due to the increased pressure developed at the liquid meniscus-air interface that tends to push the meniscus back. Figure 8 shows a pinned meniscus inside a microchannel that is pushed back due to a high airflow rate in channel $\mathrm{B}$.

Regulated airflow in the side channel (channel B) forms an important control factor for the micropump. A constant airflow rate in channel $\mathrm{B}$ helps to drive the vapor out of the system and re-condensation of the vapor within the device is hence prevented. Besides aiding enhanced evaporation 
(a) Velocity Control using Temperature/Geometry

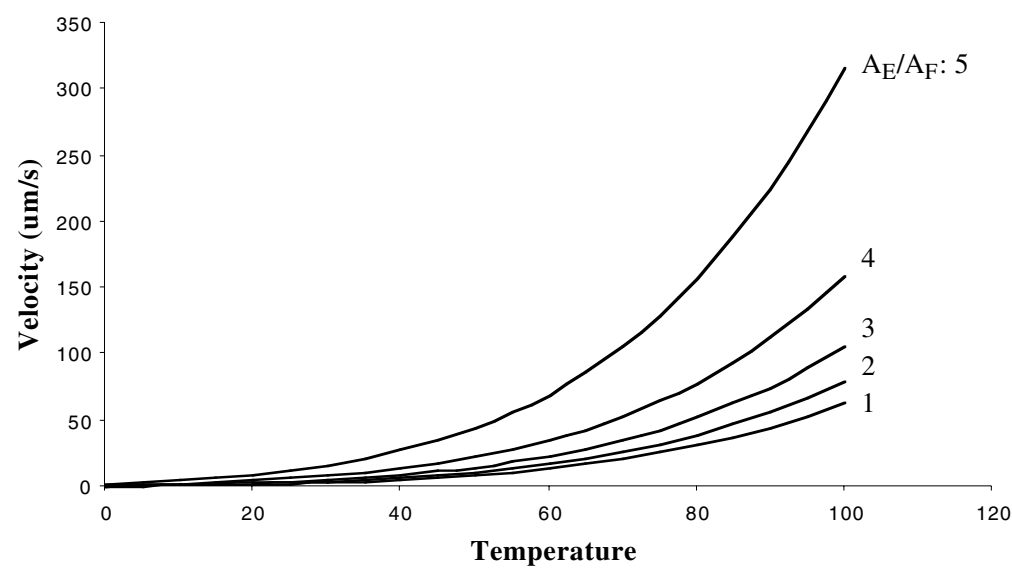

(b)

Velocity control using Temperature/Airflow

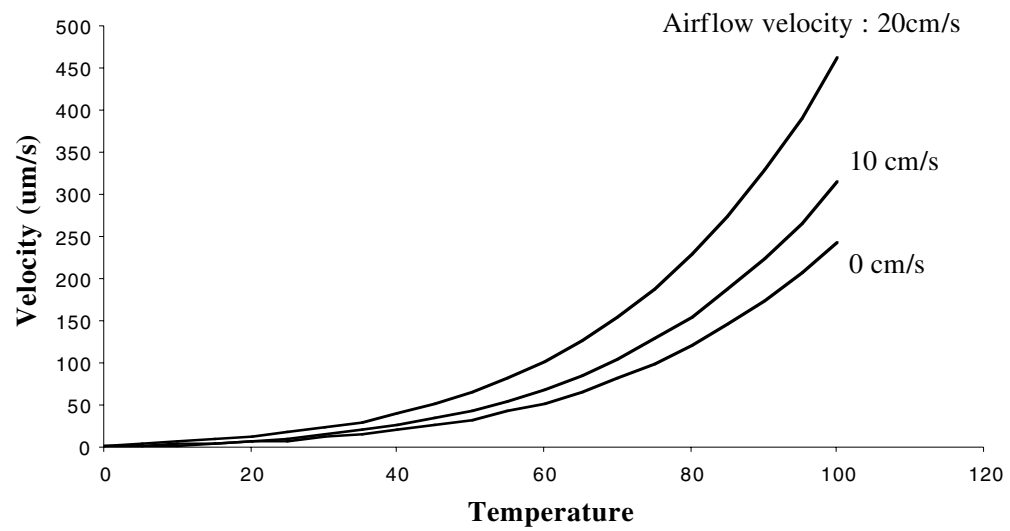

Figure 4. Model predictions. (a) Theoretically expected pumping velocities in micropumps with various cross-sectional ratios $\left(A_{E} / A_{F}\right)$. Average liquid flow velocities are plotted against the temperature at the meniscus. Water is the liquid of choice and a constant airflow rate of $10 \mathrm{~cm} \mathrm{~s}^{-1}$ is maintained in channel B. (b) Prediction of the effect of airflow on liquid pumping velocities. These results apply to a device with a cross-sectional ratio of 5 .

(a)

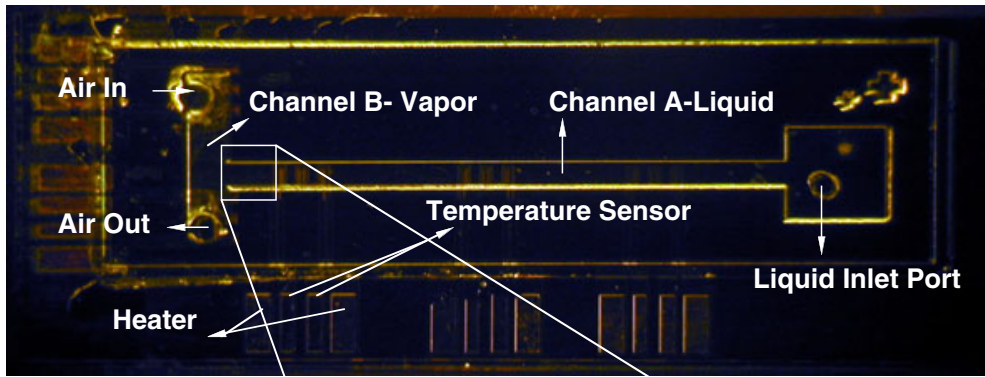

$(b)$

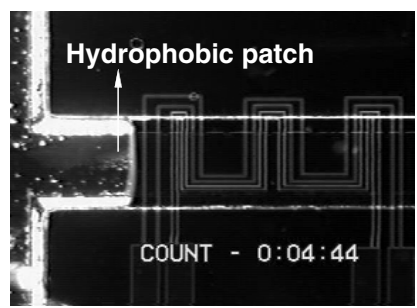

Figure 5. Illustration of the micropump operation. (a) Photograph of a fully assembled micropump. (b) Water stopped at the hydrophobic patch showing a flat meniscus. 


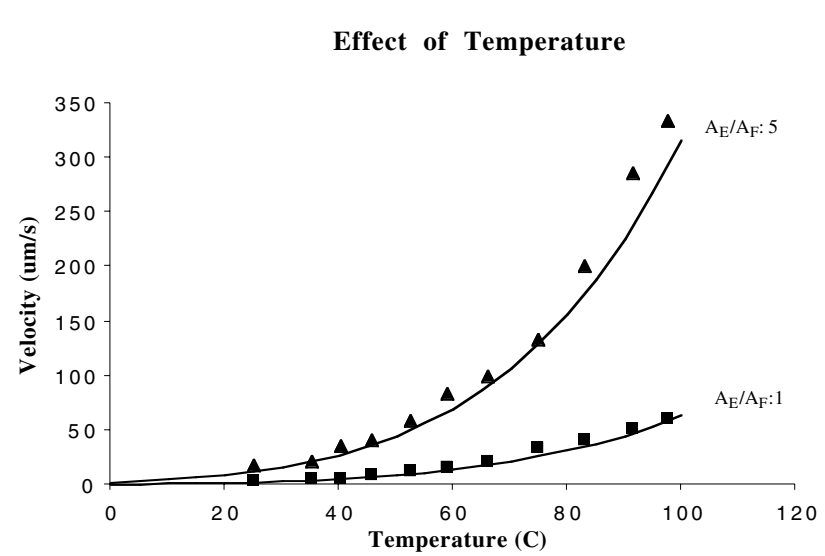

Figure 6. Experimentally determined pumping velocities of water at various operating temperatures. Two micropumps with cross-sectional ratios of 1 and 5 were tested. Constant airflow rate of $10 \mathrm{~cm} \mathrm{~s}^{-1}$ was maintained. Experimentally measured velocities are compared with the expected velocities from the model described in figure 4. Solid lines show the model values.

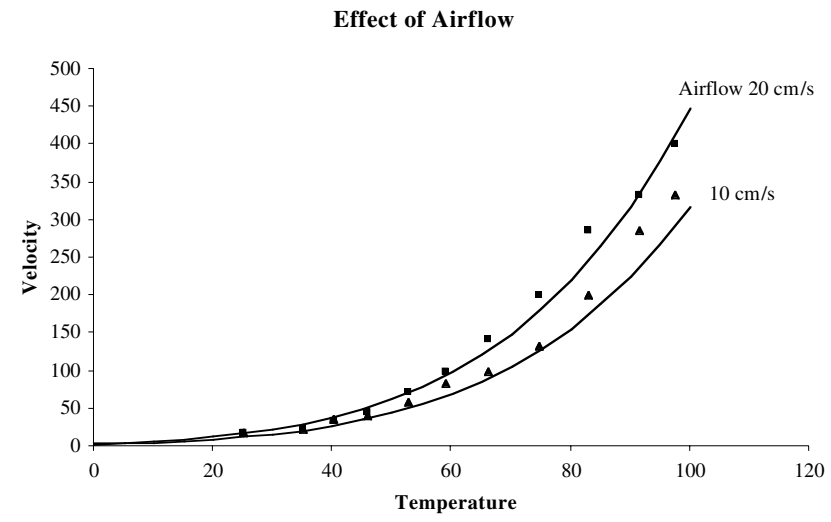

Figure 7. Effect of airflow on pumping performance. Pumping velocities of water determined at various operating temperatures for two different airflow velocities $\left(10 \mathrm{~cm} \mathrm{~s}^{-1}, 20 \mathrm{~cm} \mathrm{~s}^{-1}\right)$ in channel B. A micropump with cross-sectional ratio of 5:1 was used. Comparison of experimentally measured values with predicted values from the model is also shown. Solid lines show the model values.

from the meniscus by convection, the airflow rate also serves to maintain a constant vapor pressure in the side channel. Without the constant heated airflow in the side channel, the pumping characteristics of the micropump can vary drastically due to changes in humidity, which calls for day-to-day characterization of the pump. Another simple way to eliminate humidity changes is to use nitrogen or inert gases.

4.2.3. Device geometry. The most obvious design parameter affecting the liquid pumping velocity is the ratio of cross sections $\left(A_{E} / A_{F}\right)$. Based on the prediction from equation (8), as this ratio increases the liquid flow velocity increases in channel A. Figure 9 shows the set of devices that were assembled and tested. Velocity measurements were made in all these devices at different operating temperatures under a constant airflow rate. Figure 10 shows the experimental results of these studies for five different device geometries $\left(A_{E} / A_{F}\right.$ : (a)

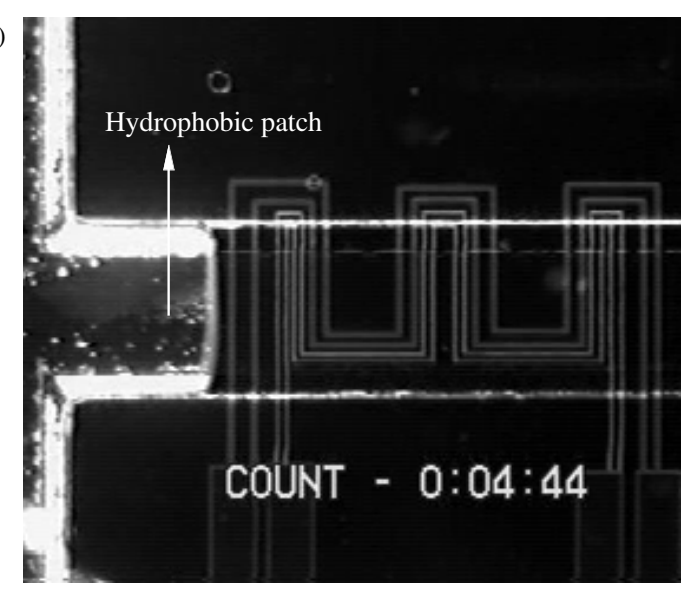

(b)

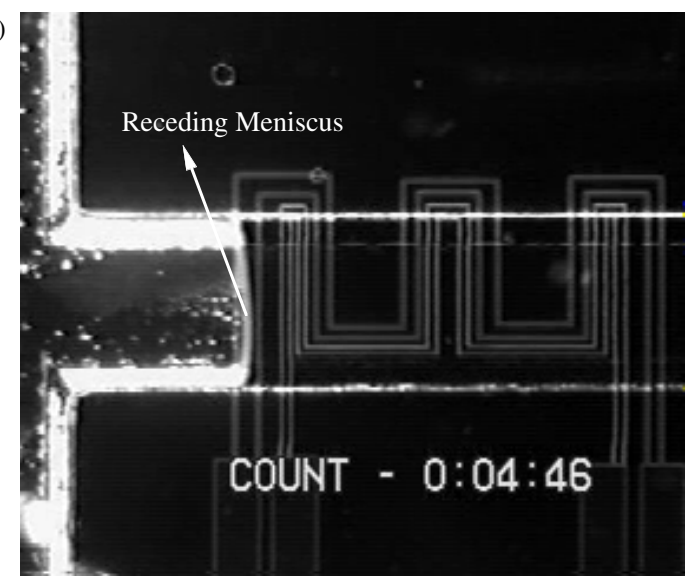

Figure 8. Effect of high $\left(>25 \mathrm{~cm} \mathrm{~s}^{-1}\right.$ ) airflow rates. (a) Water meniscus pinned at the hydrophobic patch under a constant airflow rate of $10 \mathrm{~cm} \mathrm{~s}^{-1}$. (b) Airflow rate is increased to $25 \mathrm{~cm} \mathrm{~s}^{-1}$ and the meniscus is seen to recede.

5, 4, 3, 2 and 1) along with model-predicted values for two of the device geometries $\left(A_{E} / \mathrm{A}_{\mathrm{F}}: 5\right.$ and 1$)$.

Another important design factor is the interface distance $L$. From equation (8), we can conclude that the shorter this length $L$, the higher the liquid flow velocity. However, if the design length becomes very small $(<100 \mu \mathrm{m})$, the meniscus is very close to channel B through which we pump air. At this point the meniscus is seen to be very unstable even for moderate airflows and is pushed back. The contact angle of the pinned meniscus changes due to airflow and in some cases the meniscus is seen to recede, resulting in unstable liquid flow velocities. Two different devices with varying interface lengths (150 and $300 \mu \mathrm{m}$ ) were tested. In our studies, we have seen that devices with $150 \mu \mathrm{m}$ interface lengths were unstable under external airflow and devices with $300 \mu \mathrm{m}$ worked well under moderate airflow rates. Based on the same argument, the airflow channel design (channel B) also requires a certain minimal width. Channels with very small widths $(<100 \mu \mathrm{m})$, close to the depth of the channel, have high airflow velocities coupled with a large pressure drop. Both these factors again tend to make the pinned meniscus unstable. Airflow channels ( $2 \mathrm{~mm}$ long and $700 \mu \mathrm{m}$ wide) were used for our experiments. In all these studies the depth of the channels was maintained constant at $50 \mu \mathrm{m}$. 
(a)

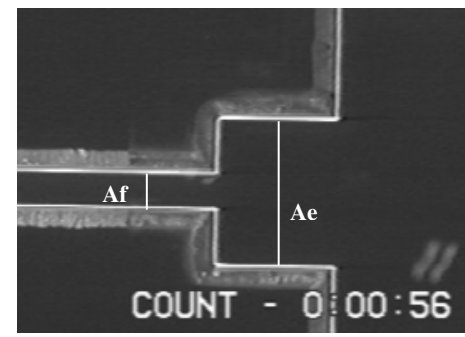

(c)

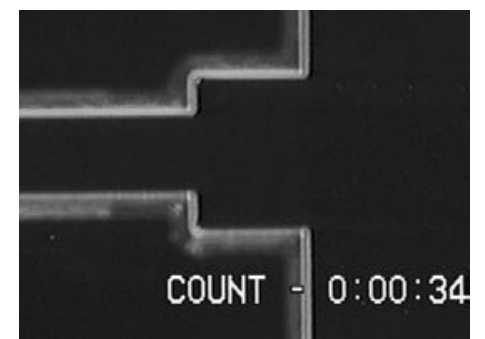

(b)

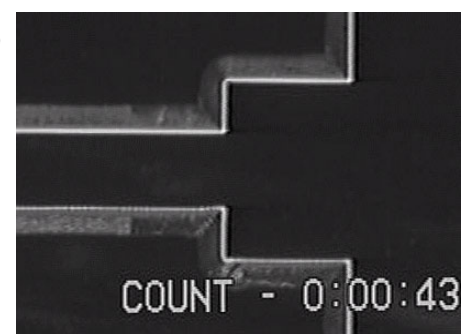

(d)

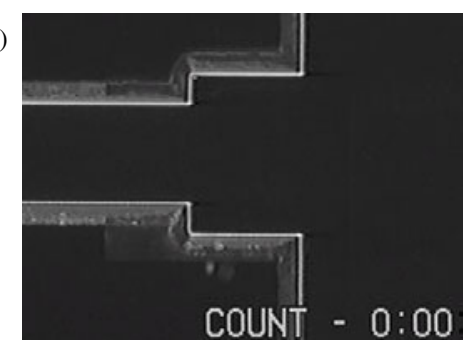

(e)

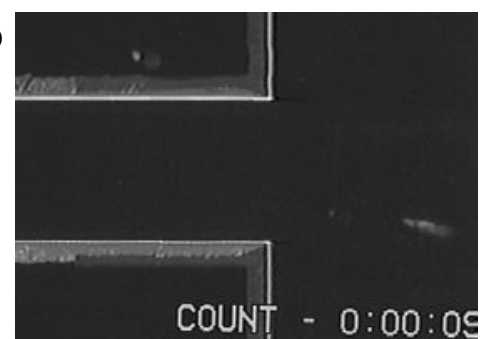

Figure 9. Zoomed-in pictures of devices with various cross-sectional ratios $\left(A_{E} / A_{F}\right) .(a)\left(A_{E} / A_{F}\right) 5: 1,(b)\left(A_{E} / A_{F}\right) 5: 2,(c)\left(A_{E} / A_{F}\right) 5: 3$, (d) $\left(A_{E} / A_{F}\right)$ 5:4, (e) $\left(A_{E} / A_{F}\right) 5: 5$.

\section{Effect of Cross-sectional Ratio}

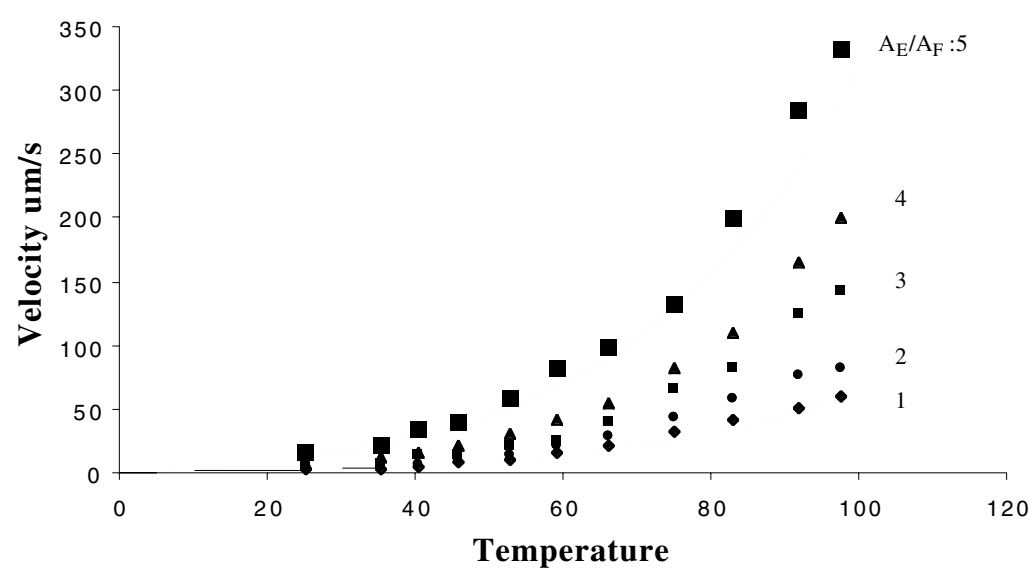

Figure 10. Pumping performance of five different micropumps (described in figure 9) with varying cross-sectional ratios $(1,1.25,1.66,2.5$ and 5) is shown. Constant airflow rate of $10 \mathrm{~cm} \mathrm{~s}^{-1}$ was maintained. The two solid lines show the model values for $\left(A_{E} / A_{F}\right)$ ratios designed at 1 and 5 .

\subsection{Time studies}

4.3.1. Response time. The response time of our pump, a measure of how long it takes to initiate a constant flow rate or stop the flow, is mainly dependent on the heat transfer characteristics of the device. The time taken to heat up the liquid meniscus to a certain desired temperature is the limiting step to initiate a constant flow in the device and similarly, the cooling-down characteristics of the device determines the stop response time. The time taken to heat distilled water from room temperature to $70{ }^{\circ} \mathrm{C}$ (using on-chip heaters), and to initiate a small constant flow rate of $\sim 40 \mathrm{nl} \mathrm{min}-1$ in a channel geometry with a cross-sectional ratio of $1: 1$ was chosen as a standard. Since our device uses silicon (thermal conductivity $=148 \mathrm{~W} / \mathrm{m} / \mathrm{K}$ ) as the substrate and on-chip heaters just behind the meniscus, fast temperature ramp-up rates of the order of $25{ }^{\circ} \mathrm{C} \mathrm{s}^{-1}$ were achieved. As a result we could initiate and maintain a constant flow rate of $40 \mathrm{nl} \mathrm{min}-1$ within $2 \mathrm{~s}$ after the power was turned on. 


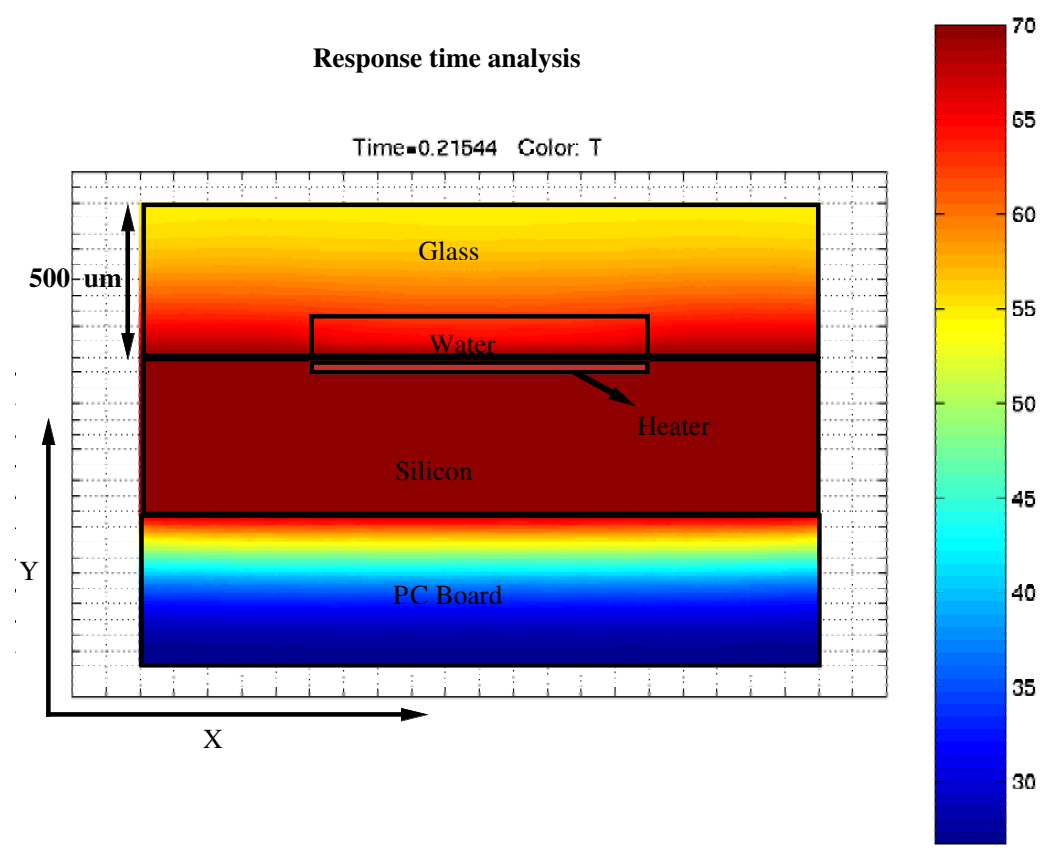

Figure 11. Dynamic heat transfer simulation of the pump showing the temperature distribution in the channel cross section above the heater. Heater is maintained at $70{ }^{\circ} \mathrm{C}$ at time $t=0$ and liquid temperature in the channel reaches $65{ }^{\circ} \mathrm{C}$ after $214 \mathrm{~ms}$.

To verify this experimental observation, dynamic heat transfer simulations of the system were done using a commercial finite element analysis software (MATLABPDETOOL). Figure 11 shows the simulated temperature profile in the device, $214 \mathrm{~ms}$ after the heater was turned on. The cross section of the device with all the materials is shown. For this study, the heater was set at $70{ }^{\circ} \mathrm{C}$ at time $t=0$ and the boundary conditions (at the edges of the device) were set to cooling by convection to the ambient. If the heater is maintained at a constant temperature of $70{ }^{\circ} \mathrm{C}$ at time $t=0$, then water in the microchannel can theoretically reach $70^{\circ} \mathrm{C}$ just after $214 \mathrm{~ms}$ as shown. To study the stop-response time, the heater was turned off and the time taken for the flow to stop when the device cools down to room temperature was measured. To accelerate the temperature ramp down, a peltier device maintained at $5{ }^{\circ} \mathrm{C}$ was placed under the micropump during the cooling-down stage. The observed stop-response time was about $4 \mathrm{~s}$.

4.3.2. Robustness. One way to characterize the robustness of the micropump is by studying how long a constant flow rate could be sustained. For this study again a constant flow rate of $35 \mathrm{nl} \mathrm{min}{ }^{-1}$ was initiated in a micropump with a geometry ratio of 1:1. The temperature of the meniscus was maintained at $65^{\circ} \mathrm{C}$ and the airflow rate was held constant at $10 \mathrm{~cm} \mathrm{~s}^{-1}$. The flow rate was monitored by measuring the velocity of fluorescein microspheres at constant intervals of $5 \mathrm{~min}$. Figure 12 shows the continuous flow rate maintained by the pump over $2 \mathrm{~h}$ of continuous operation. The decrease in flow rate after $2 \mathrm{~h}$ of operation is possibly because of the accumulation of the fluorescein microspheres at the liquid meniscus.

Continuous working of the micropump also depends on the amount of liquid head held at the reservoir end, which in turn depends on how much differential pressure the

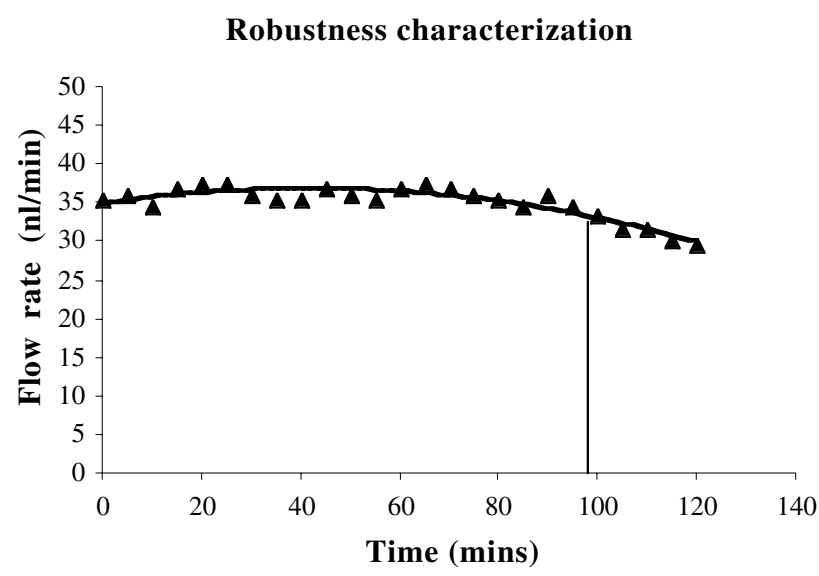

Figure 12. Pumping performance of the device characterized over a period of $2 \mathrm{~h}$. A micropump with $\left(A_{E} / A_{F}\right)$ 1:1 working under a constant airflow rate of $10 \mathrm{~cm} \mathrm{~s}^{-1}$ was used.

hydrophobic patch at the meniscus end can withstand. Earlier experiments in our group have shown that we can support up to a $2^{\prime \prime}$ water head using the hydrophobic patches. Using a $2^{\prime \prime}$ water head at the reservoir end inside a $350 \mu \mathrm{m}$ radius capillary (as described in section 4.1) translates to about $20 \mu \mathrm{l}$ water in that cylindrical reservoir. This implies continuous pumping for about $10 \mathrm{~h}$ at a constant pumping rate of $30 \mathrm{nl} \mathrm{min}-1$, provided that ideal operating conditions can be achieved.

\section{Summary and conclusions}

We have developed a novel micropump to deliver nonpulsatile, ultra-low flow velocities in the $100 \mu \mathrm{m} \mathrm{s}^{-1}$ range. The pumping mechanism is based on the concept of evaporation of liquid at a pinned meniscus and capillarity action in microchannels. Resulting flow rates 


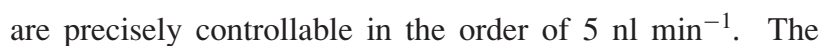
micropump is fabricated using the well-characterized silicon microfabrication techniques, does not involve any moving parts and hence is found to be very robust in operation.

Our transpiration-based micropump, though demonstrated for pumping water, can be potentially used for pumping most solvents with viscosities similar to water. However, this will require modifications in the hydrophobic patch design (to maintain a flat meniscus). The micropump may be of use in several emerging biological studies including stretching single DNA molecules, monitoring cell adhesion and understanding shear effects on immobilized proteins. The pumping concept can be further extended to include several lanes, each of which has a pinned meniscus. By controlling the evaporation rate at a meniscus in one lane, we can expect to see some interesting flow-direction-switching behavior. Such a device can also be used for sorting molecules. On the other hand, a device with several reservoirs and evaporation at a single pinned meniscus can be used to create hydrodynamic focusing effects.

\section{Acknowledgments}

This work was supported by the National Institutes of Health under grants NIH-R01-HG01044 and NIH-R01-HG01406. We are thankful to Kalyan Handique, Vinod A Suresh and Dylan Heldsinger for their help.

\section{References}

Bird R et al 1960 Transport Phenomena (New York: Wiley)

Burns M A et al 1998 An integrated nanoliter DNA analysis device Science 282 484-7

Canny M 1998 Applications of the compensating pressure theory of water transport Am. J. Botany 85 897-909

Carrozza M C et al 1995 A piezoelectric-driven stereolithography-fabricated micropump J. Micromech. Microeng. 5 177-9

Cussler E L 1997 Diffusion (Cambridge: Cambridge University Press)

Davenport R J et al 2000 Single molecule study of transcriptional pause and arrest by E. coli RNA polymerase Science $\mathbf{2 8 7}$ 2497-500

Dorre K 1997 Techniques for single molecule sequencing Bioimaging 5 139-52

Fu A Y et al 1999 A microfabricated fluorescence-activated cell sorter Nature Biotechnol. 17 1109-11
Goedecke N and Manz A 2001 Towards evaporation-driven HPLC on a chip: an alternative transport process for microanalysis systems MicroTAS 2001 (Monterey, CA)

Handique K et al 2000 Nanoliter liquid metering in microchannels using hydrophobic patterns Anal. Chem. 72 4100-9

Jing J et al 1998 Automated high resolution optical mapping using arrayed, fluid-fixed DNA molecules Proc National Academy of Sciences vol 95 pp 8046-51

Liu S et al 1999 Optimization of high-speed DNA sequencing on microfabricated capillary electrophoresis channels Anal. Chem 71 566-73

Manz A et al 1995 Electroosmotic pumping and electrophoretic separations for miniaturized chemical analysis systems $J$. Micromech. Microeng. 4257

Mehta A D et al 1999 Myosin-V is a processive actin-based motor Nature 400 590-3

Namasivayam V et al 2000 A microfabricated valveless pump for delivering non-pulsatile flow Proc. SPIE: Micromachining, Micromanufacturing and Microelectronic Manufacturing (Santa Clara, $C A$ )

Olsson A E P et al 1997 Micromachined flat-walled valveless diffuser pumps J. Microelectromech. Syst. 6 161-6

Perkins T T et al 1995 Stretching of a single tethered polymer in a uniform flow Science 268 83-87

Salas-Solano O et al 2000 Optimization of high-performance DNA sequencing on short microfabricated electrophoretic devices Anal. Chem. 72 3129-37

Schonberg J A et al 1995 An augmented Young-Laplace model of an evaporating meniscus in a microchannel with a high heat flux Exp. Therm. Fluid Sci. 10 163-70

Shoji S and Esahi M 1990 Technical Digest of the 9th Sensor Symposium pp 27-30

Smith J and Van Ness H 1987 Introduction to Chemical Engineering Thermodynamics (New York: McGraw-Hill)

Srinivasan U et al 1997 Self-assembled fluorocarbon films for enhanced stiction reduction Transducers 97, Int. Conf. Solid-State Sensors and Actuators vol 2 (New York: Institute of Electrical and Electronics Engineers) pp 1399-402

Takayama S et al 2001 Laminar flows: subcellular positioning of small molecules Nature 4111016

Vand De Pol F C M et al 1989 A thermo-pneumatic actuation principle for a microminiature pump and other micromechanical devices Sensors Actuators A 17 139-43

Waters L C et al 1998 Microchip device for cell lysis, multiplex PCR amplification, and electrophoretic sizing Anal. Chem. 70 158-62

Woolley A T et al 1997 High-speed DNA genotyping using microfabricated capillary array electrophoresis chips Anal. Chem. $692181-6$

Zengerle A et al 1992 A micro membrane pump with electrostatic actuation Proc. IEEE MEMS (Travemunde, Germany) pp 19-23 